\title{
Patient Loyalty in a Mature IDS Market: Is Population Health Management Worth It?
}

\author{
Caroline S. Carlin
}

Objective. To understand patient loyalty to providers over time, informing effective population health management.

Study Setting. Patient care-seeking patterns over a 6-year timeframe in Minnesota, where care systems have a significant portion of their revenue generated by shared-saving contracts with public and private payers.

Study Design. Weibull duration and probit models were used to examine patterns of patient attribution to a care system and the continuity of patient affiliation with a care system. Clustering of errors within family unit was used to account for within-family correlation in unobserved characteristics that affect patient loyalty.

Data Collection. The payer provided data from health plan administrative files, matched to U.S. Census-based characteristics of the patient's neighborhood. Patients were retrospectively attributed to health care systems based on patterns of primary care.

Principal Findings. I find significant patient loyalty, with past loyalty a very strong predictor of future relationship. Relationships were shorter when the patient's health status was complex and when the patient's care system was smaller.

Conclusions. Population health management can be beneficial to the care system making this investment, particularly for patients exhibiting prior continuity in care system choice. The results suggest that co-located primary and specialty services are important in maintaining primary care loyalty.

Key Words. Consumer issues, physician payment, organization and delivery of care

Health care in the United States is in the midst of change, as we refocus attention from treatment of acute care to population health management, supported by a shift in provider reimbursement from fee-for-service payment to payment methods that incorporate measures of total cost of care. The Medicare Shared Savings Program (Centers for Medicare and Medicaid Services 2013) and similar shared savings arrangements used by private 
payers (Bailit et al. 2012) are examples of this shift in reimbursement mechanism. Because our health care culture has historically emphasized acute care, the new emphasis on population health management requires significant structural and financial changes for these care systems. Use of the term "care system" encompasses physician-only clinic systems and integrated delivery systems (IDSs) capable of contracting as Accountable Care Organizations (ACOs).

Uncertainty is generated by the time that elapses between health management activities (e.g., better cholesterol control) and emergence of savings in acute care (e.g., reduced costs for cardiac events). If there is significant turnover in patient population, the care system may never reap the financial benefit of investing in population health. ${ }^{1}$ In addition, because a shared savings program typically computes total-cost-of-care measures based on a patient population retrospectively attributed to the care system based on patterns of care, there is uncertainty in predicting the population for which the care system will be held accountable.

This work, motivated by questions from the provider community, attempts to inform population health management activities under shared savings contracts. Specifically, I considered the following questions:

- Which health plan members are most likely to be attributed to a care system?

- Once a member has been attributed to a care system, what is the likelihood he or she will switch to another system in the future?

- What is the expected length of time a member will use a specific care system as his or her primary source of care?

I examined these questions in the context of Minnesota, a market characterized by large, vertically integrated delivery systems (IDSs). Because Minnesota is on the leading edge of national trends in provider consolidation and shared savings contracting, we believe this work will be informative nationally as other provider communities begin to shift their focus to population health management. The strong patient loyalty found, enhanced for vertically integrated delivery systems most likely to participate in shared savings contracts, means that these population health management activities benefit the contracting care system as well as the patient. 


\section{BACKGROUND}

\section{Continuity of Patient Relationship with a Care System}

While there is a significant body of research examining the importance of continuity in patient relationships with providers, much of that literature focuses on continuity between patients and individual providers. I focus on longitudinal continuity between patient and the provider care system (measured by patterns of primary care access), which is linked to the important dimensions of continuity in information and interpersonal continuity between the patient and individual physicians (Saultz 2003; Waibel et al. 2012).

The majority of the literature examining factors that predict continuity of primary care relationships is based in settings outside of the United States and relies on patient self-report of continuity, although this literature consistently finds increased continuity of primary care for those who are female, older, have (more) chronic diseases, and lower education levels (Guthrie 2002; Mendoza-Sassi and Beria 2003; Rosa Filho, Fassa, and Paniz 2008; Lee et al. 2013).

\section{Retrospective Attribution}

Because many payer-provider shared savings contracts involve open-access insurance design (no prospectively selected "medical home" functioning as a gatekeeper), members are usually attributed to care systems retrospectively. Under a typical shared saving contract (Morris and Eggbeer 2011; Bailit et al. 2012), the plan pays the care system on a fee-for-service basis during the contract year. At the end of the year, members are attributed to the care system based on their patterns of care, and the risk-adjusted total cost of care delivered is compared with a target cost. If the actual cost is lower than the target, the care system and payer share this savings. These contracts are relatively new, and retrospective attribution methods vary (Mehrotra et al. 2010; Bailit et al. 2012).

\section{Study Setting}

This work is unique in its focus on primary care continuity measures germane to shared savings programs, such as Medicare's Shared Savings Programs and their Pioneer ACO program, and the shared savings programs that are increasingly used for provider contracting by private payers. I examined the population enrolled in a regional health plan, Medica, headquartered in the 
Minneapolis-St. Paul area. Medica entered into its first shared savings contract in 2009, and this contracting method has spread across payers and care systems to become the most prevalent contracting method in Minnesota between private payers and vertically integrated (hospital and physician) delivery systems capable of functioning as an ACO (Christianson et al. 2011). Historically, Medica has used retrospective attribution to develop the health plan member population for whom each care system is responsible under the shared saving contract. Each member in Medica's commercial and managed Medicaid populations was attributed to a care system if they received 50 percent or more of their primary care services from that care system. ${ }^{2}$ I used this retrospective attribution methodology applied by the plan to the member population residing in the state of Minnesota in the years 2006 through 2011 to observe how the member populations changed from the perspective of the care system across time. The member panel can change because the member switched the care system from which he or she received the majority of primary care, because the member did not seek primary care or did not reach the 50 percent benchmark for any care system in the year, or because he or she disenrolled from coverage with Medica. By understanding how stable these relationships between patients and care systems are, we gain understanding of the potential for long-term population health management, and we gain understanding of the care system's ability to recapture their investment in population health through reduced future utilization generated by the improvement in patient health.

\section{DATA}

We pulled enrollment data for the years 2006 through 2011 for all health plan members covered by a commercial group, individual, or managed Medicaid product for residents in Minnesota. Table 1 summarizes the total observations available and the observations used in each of the baseline models. These enrollment data tell us the member's age and gender, months of coverage, and the plan in which he or she was enrolled. Our population had an average age of 30 and was 52 percent female. Sixty-one percent of the population was enrolled in a commercial group product with a broad PPO network, and 10 percent in a commercial group product with a tighter network requiring prospective medical home designation, 3 percent in individual commercial coverage, and the remaining 25 percent in a managed Medicaid product. I have excluded from the data infants and those ages 65 or older. The infant results 


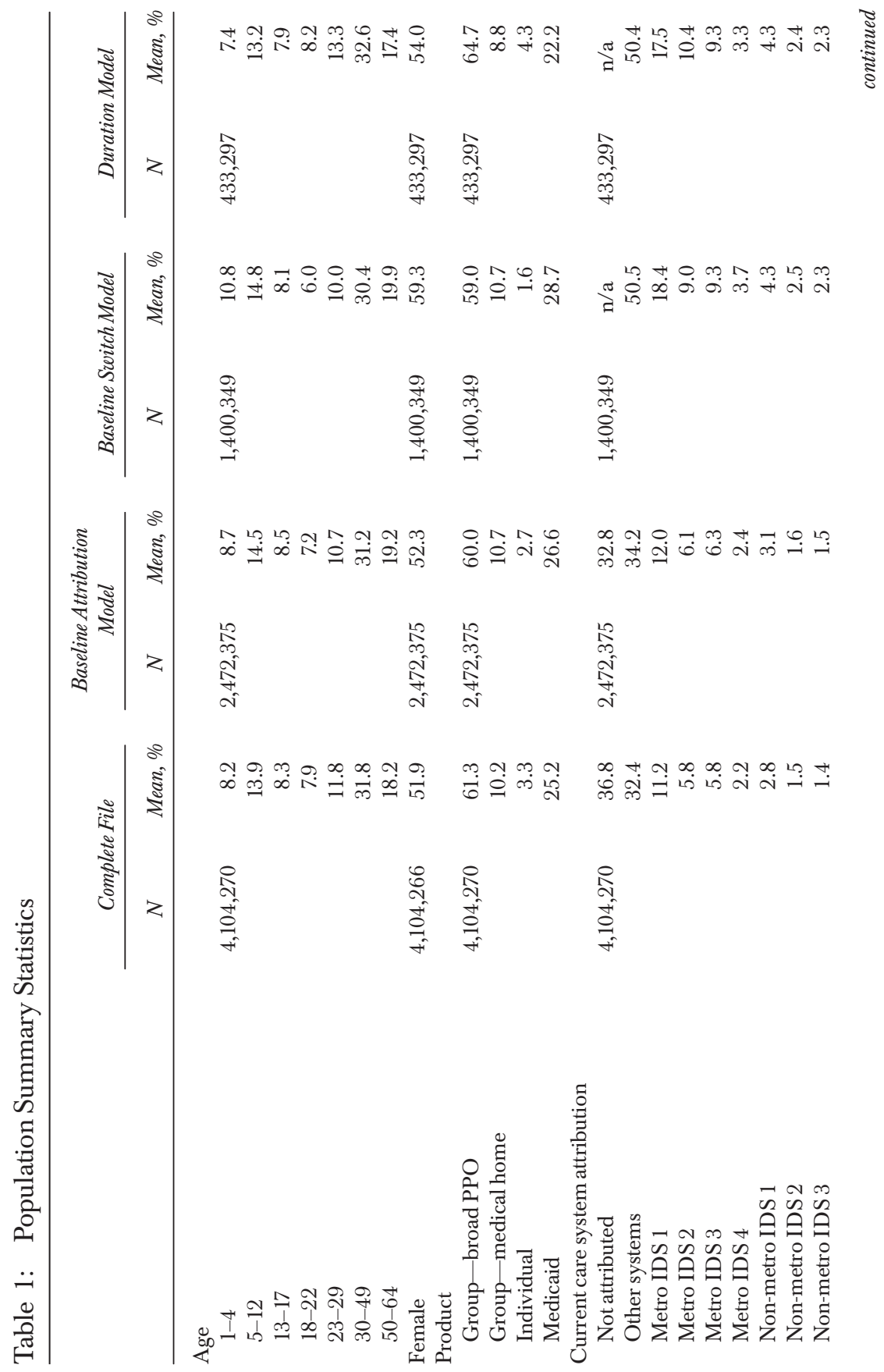




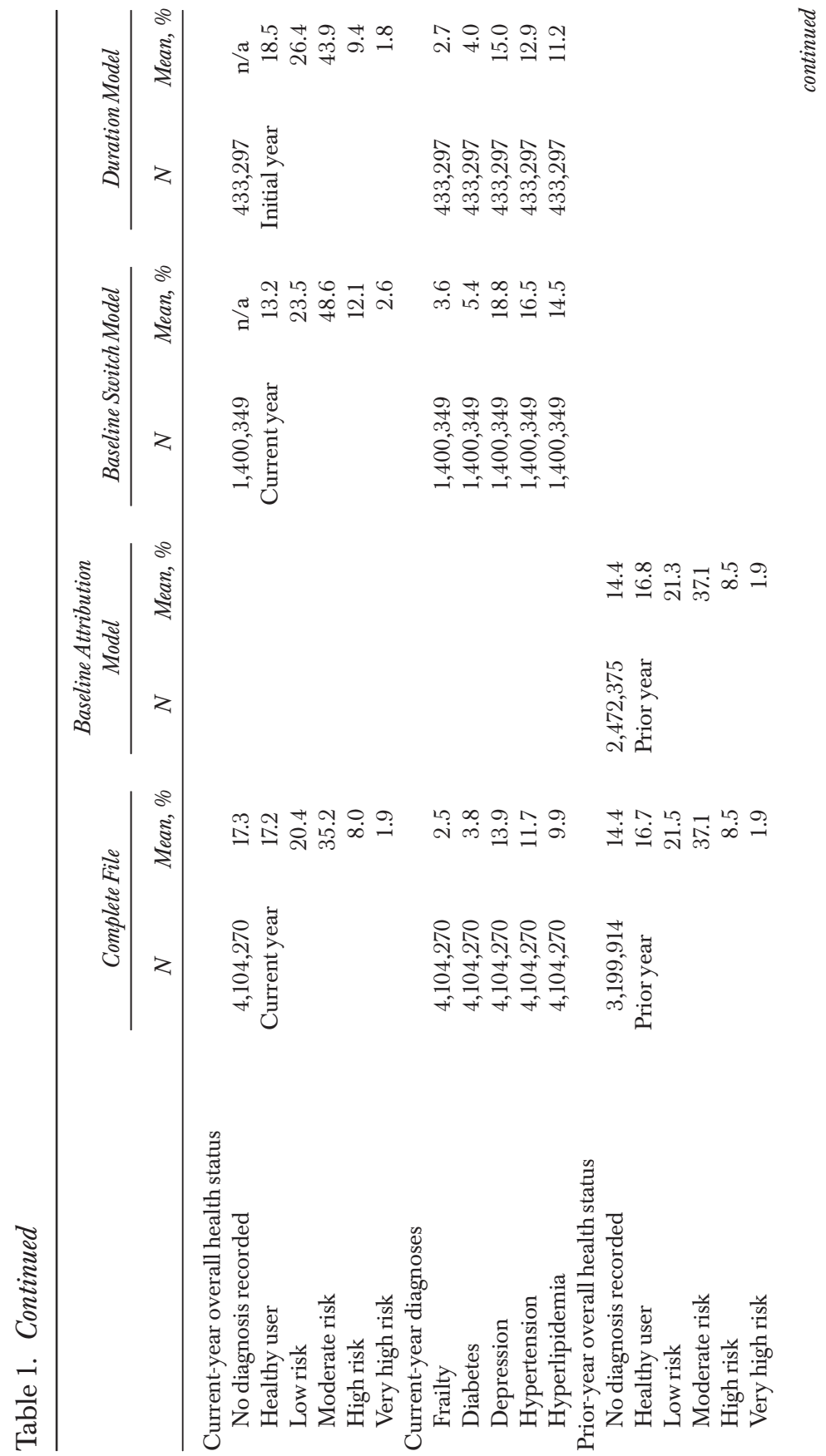




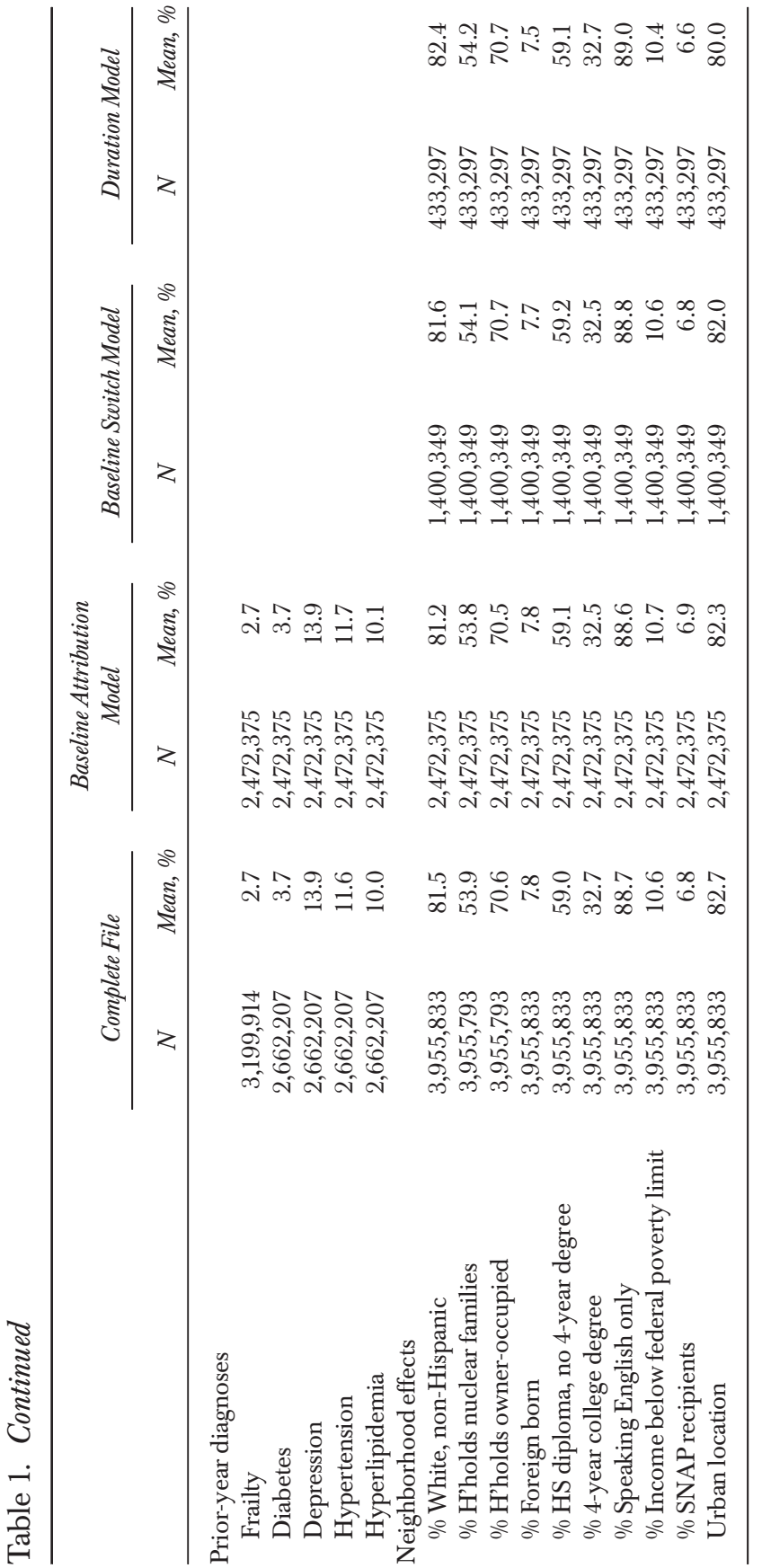


were distorted by a concentration of Medicaid enrollment; the over-65 population was small and atypical due to the interaction between Medicare coordination rules and active employment.

To this enrollment data we added two sets of claims-based variables. The first set of variables, most critical to this analysis, is the member attribution to a care system. Of the person-years of enrollment captured, 63 percent had accessed primary care in a way that met Medica's criteria for attribution to a care system. Of those attributed, there is significant concentration by care system, with nearly half ( 49 percent) attributed to one of the top seven IDSs in the state; four are headquartered in the Minneapolis-St. Paul metropolitan area ("Metro IDS"), and three are headquartered in other communities ("Non-metro IDS").

The second set of claims-based variables are measures of health risk developed using the Johns Hopkins ACG system (Weiner, Starfield, and Lieberman 1992). This system uses diagnosis code history to create a categorical variable classifying each member into a resource utilization band: "No valid diagnosis" (primarily those who never accessed care), "healthy users," "low health risk," "moderate health risk," "high health risk," and "very high health risk." In addition, I used binary variables indicating frailty or the presence of the four most prevalent chronic conditions: diabetes, hypertension, hyperlipidemia, and depression. Health risk measures are summarized for the person-years observed, as well as for the prior years, when available.

Finally, the informatics team geocoded each member's address and matched to variables capturing neighborhood effects expected to impact the stability of patient affiliation with a care system. From the 2010 U.S. Census, measured at block level, we used race and ethnicity (percent white, non-Hispanic). At the block-group level, we used the following measures from the 2011 5-year average American Community Survey (U.S. Census Bureau 2012) statistics: percent housing units owner-occupied, and percent households that are nuclear families. At the census-tract level, we used the following measures from the 20115 -year average American Community Survey statistics: percent foreign born, percent speaking English only, percent with income below federal poverty limit, percent households using SNAP (food stamps) in the past year, education level (percent with high school degree but no 4-year college degree, percent with 4-year college degree). Finally, we categorized the residence as "Urban" if the Rural Urban Commuting Area code (WWAMI Rural Health Research Center 2013) was in the "Urban focused" list (1.0, 1.1, 2.0, 2.1, 3.0, 4.1, 5.1, 7.1, 8.1, 10.1). Once these geocoded matches were 
complete, all explicit identifiers were removed from the database to create a limited dataset, prior to delivery to the researcher.

\section{ECONOMETRIC FRAMEWORK}

\section{Which Members Are Most Likely to Be Attributed to a Care System?}

I used a simple probit equation to model the probability of being attributed to a care system in any 1 year of enrollment, with clustering of errors within the family unit to account for within-family correlation in propensity to be attributed. I modeled the probability the individual was attributed in the current year, based on current age, gender, plan enrollment, and neighborhood effects, and based on the prior year's health status to avoid having current health status confound current patterns of care-seeking. I have $N=2,472,375$ person-year observations for which I can obtain prior-year health status. The subset of observations for which I can observe prior-year information has a somewhat worse health status and attribution rate (66 percent vs. 62 percent in the full population). Because of this change in population, in Table 2 I display results of model estimation with and without prior-year health status and diagnosis information as a robustness check.

\section{What Is the Probability That a Member Will Switch Care Systems?}

I used a probit model for $P$ (switch care system), again with clustering of errors within the family unit. Here, I used the $N=1,400,349$ person-years for individuals currently attributed, for whom I observed a future year of attribution. The binary "switch" variable was defined by comparing the current and future attribution. Using current age, gender, plan enrollment, health status, and neighborhood effects, I modeled the future "switch" decision. Because I have current attribution, I could also include a categorical variable indicating the current care system to which the individual was attributed, allowing us to see whether patient loyalty varies by care system. I identified attribution to one of the top four metro and top three non-metro IDSs, with "other" as the reference category.

I included a second switching model, restricting the population to the $N=785,501$ observations that allow us to see three sequential attributions (prior, current, future), so that I could create a variable capturing their prior switch decision to help predict the future decision. I expected that, if they were loyal to their current care system the last time they had an opportunity to 


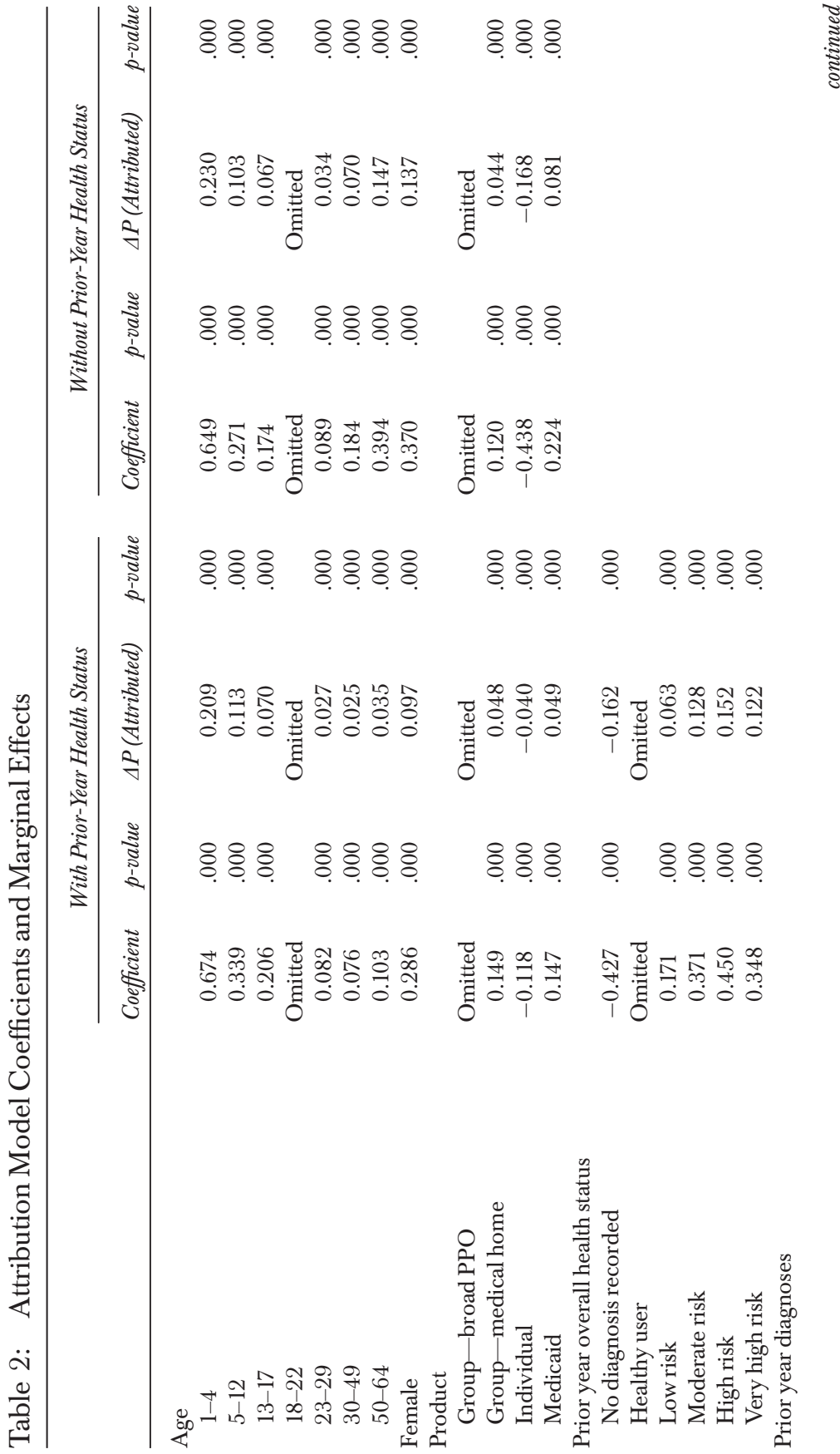




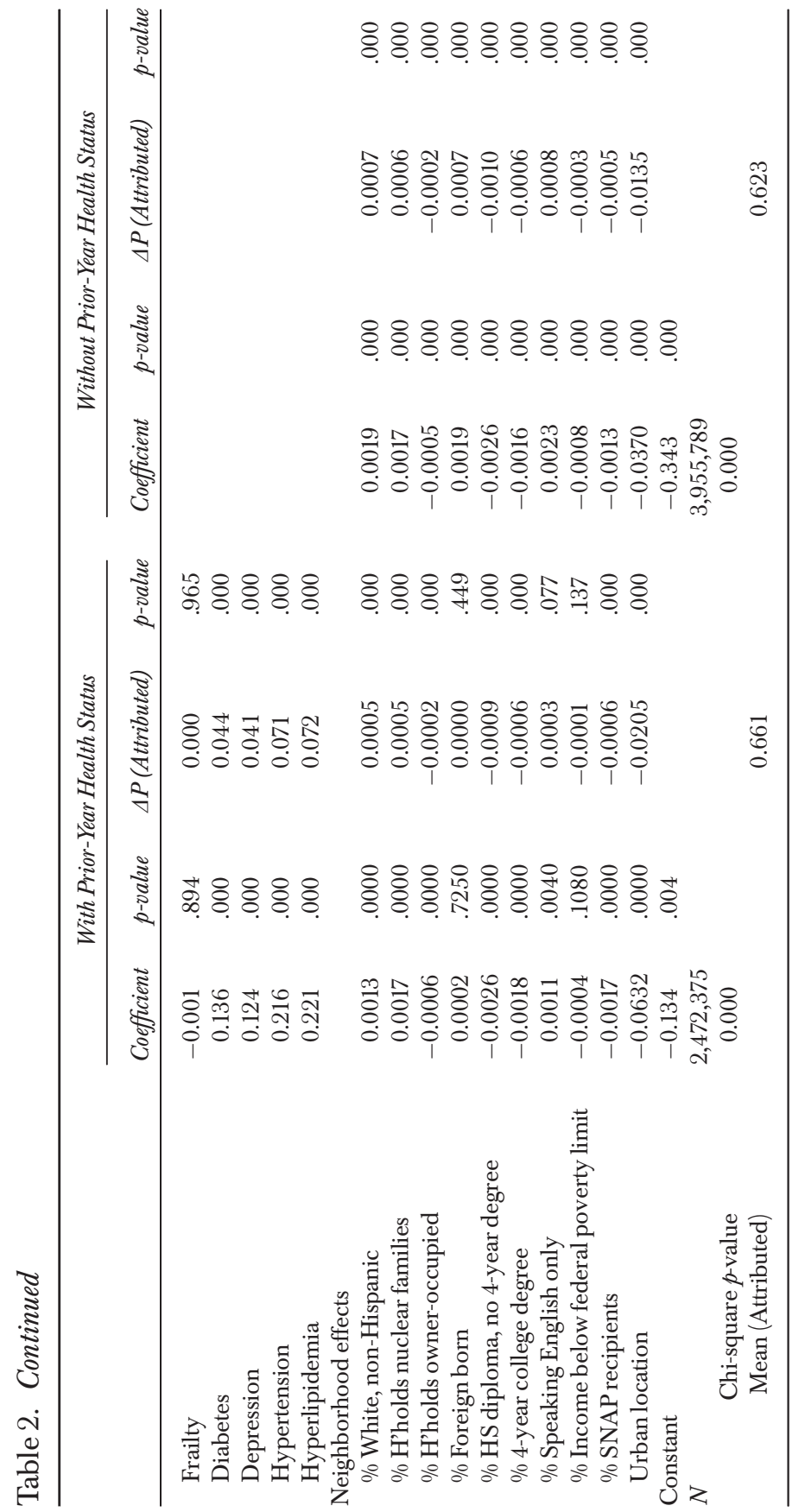


switch affiliation, they would be less likely to switch at the next opportunity. Because a member must have had at least 3 years of health plan enrollment to be included, we must acknowledge that this is probably a more stable group than the larger dataset; indeed, the average rate of switching declined slightly from 17.6 to 16.7 percent when I moved to this smaller population.

\section{What Is the Expected Duration of the Patient's Relationship with a Care System?}

This relatively long 6-year panel tracing primary care relationships over time allowed me to estimate a duration model to use in predicting the length of primary care relationships. The data are lost to observation ("censored") at the end of the 6-year observation period, or loss of Medica coverage. I used the fully parametric Weibull duration model to take this censoring into account, and to facilitate computation of expected duration of the patient relationship with a care system, and the marginal effect of member and care system characteristics on that expected duration. The population used to estimate the model included those who were newly enrolled and attributed plan members in 2006 or later $(N=433,297)$. Individuals were followed from the first year of attribution until they switched attribution, or were lost to observation, whichever occurs first. In this estimation, I allowed clustering of errors within the family group to account for within-family correlation in patient affiliation with a care system.

The individual characteristics used to model duration were measured at the time of the member's first observed attribution, and they included age, gender, insurance product, health status, initial attributed care system, and neighborhood effects. The initial attributed care system was of particular interest, as there may be characteristics of the care system that impacted patient loyalty.

\section{RESULTS}

\section{Probability of Attribution to a Care System}

The results of model estimation for the probability of attribution can be seen in Table 2, with and without prior-year health status and diagnosis information as predictors. Within each set of model results, the first two columns display the coefficients ( $p$-values) in the estimated probit model; the second two columns display the marginal effects ( $p$-values) of each variable. Here, marginal effects were defined as the percentage-point change in the 
probability of being attributed due to a one-unit change in the percentagebased neighborhood effects, or by a change in binary and categorical variables from the reference value to the value in question. For example, moving from the reference college-age years (ages 18-22) to late middle age (ages 50-64) caused a 3.5 percentage-point increase in the probability of being attributed. It is helpful to note that this marginal effect is relative to the sample's average 66.1 percent probability of attribution. We see a generally u-shaped curve for age, with the youngest ages having a significantly higher chance of attribution, and smaller increases at older age ranges. This is consistent with the frequent wellness visits expected for young children, and the steady increase in visits due to chronic diseases in later adulthood.

Relative to the broad PPO network, we see increased probabilities of being attributed for the medical home ( +4.8 percent) and the Medicaid $(+4.9$ percent $)$ products, with less attribution in the individual market $(-4.0$ percent). There is a nearly monotonic increase in the probability of attribution across the health status categories: those with no diagnosis recorded had a -16.3 percentage point lower probability of attribution relative to healthy users, while those with moderate health risk or greater had a +12 to -15 percentage point increase in probability of attribution. The presence of each chronic disease increased the probability of attribution (marginal effects 4.1-7.2 percent), but frailty alone did not appear to have an effect. While neighborhood effects are often statistically significant, the practical significance is small. For example, a 10 percentage-point increase in the percent white, non-Hispanic on the member's block caused only a +0.5 percentage-point increase in the probability of being attributed to a care system. Urban location is the only neighborhood effect approaching practical significance with a -2.1 percent marginal effect relative to rural members.

When I excluded prior-year health status and diagnosis information, much of their effect was captured in the adult age categories, due to the correlation between age and health status. The marginal effects of age increased from +3.5 percentage points for ages $50-64$ in the original model, to +14.7 percentage points in the model without prior-year health status. This change in sample had a significant impact on the effect of product; by restricting the population to those for whom we can observe a prior year, I have captured an unusually stable individually enrolled commercial population. Therefore, the impact of being enrolled in an individual product in the full-population estimation was significantly larger than the subset with prior-year health status information; the magnitude of the marginal effect increased to -16.8 percentage points from the original -4.0 . 


\section{Probability of Srwitching Care Systems}

In Table 3 I show the models estimating the probability of switching care system affiliation at the next observed attribution, with marginal effects showing the percentage-point change in the probability of switching due to each variable. Note that marginal effects are relative to an average 17.6 percent probability of switching in the population used for the first model. Consistent with all the life changes that happen in early adulthood, and the movement from pediatric to adult care, we see that ages 18-22 had the highest probability of switching care systems. The younger and older categories had significantly smaller probabilities of switching; marginal effects steadily decrease at the endpoints to -16.4 percent (ages 1-4) and -11.7 percent (ages 50-64). Females had a slightly greater probability of switching $(+1.5$ percent), counter to expectations.

Relative to those enrolled in the broad PPO product, those who elected the medical home product were more likely to stay with their original care system ( -4.2 percent); the more transient Medicaid population was more likely to switch affiliation (+3.3 percent). Those enrolled in the individual products were less likely to switch ( -0.6 percent), which is counterintuitive. Individual coverage is often bought during times of employment transition, and we would expect this period of transition to be reflected in low care system affiliation. Because I must have two successive observations to determine switching behavior, I most likely had an unusually stable segment of the individual product population included here. Indeed, this is supported by the duration results discussed below. The impact of health status on the probability of switching became meaningful for those who had high (+2.6 percent) and very high (+6.4 percent) health risk. This is counter to expectations, as the literature shows that those with a high frequency of visits place greater value on continuity of care (Nutting et al. 2003; Mohammed Al-Azri and Ganguly 2009; Gallagher et al. 2013). Those with diabetes, hypertension, and hyperlipidemia were less likely to switch care systems ( -1.5 to -4.2 percent), but those who were frail were slightly more likely to switch $(+1.8$ percent).

One of the most interesting results is the impact of care system size. Current attribution to a large IDS was associated with a much smaller probability of switching care systems ( -5.7 to -9.9 percent), with the strength of the marginal effect correlated with the relative size of the IDS.

Although often statistically significant, neighborhood effects had no practical effect on the probability of switching care systems. This is surprising, 


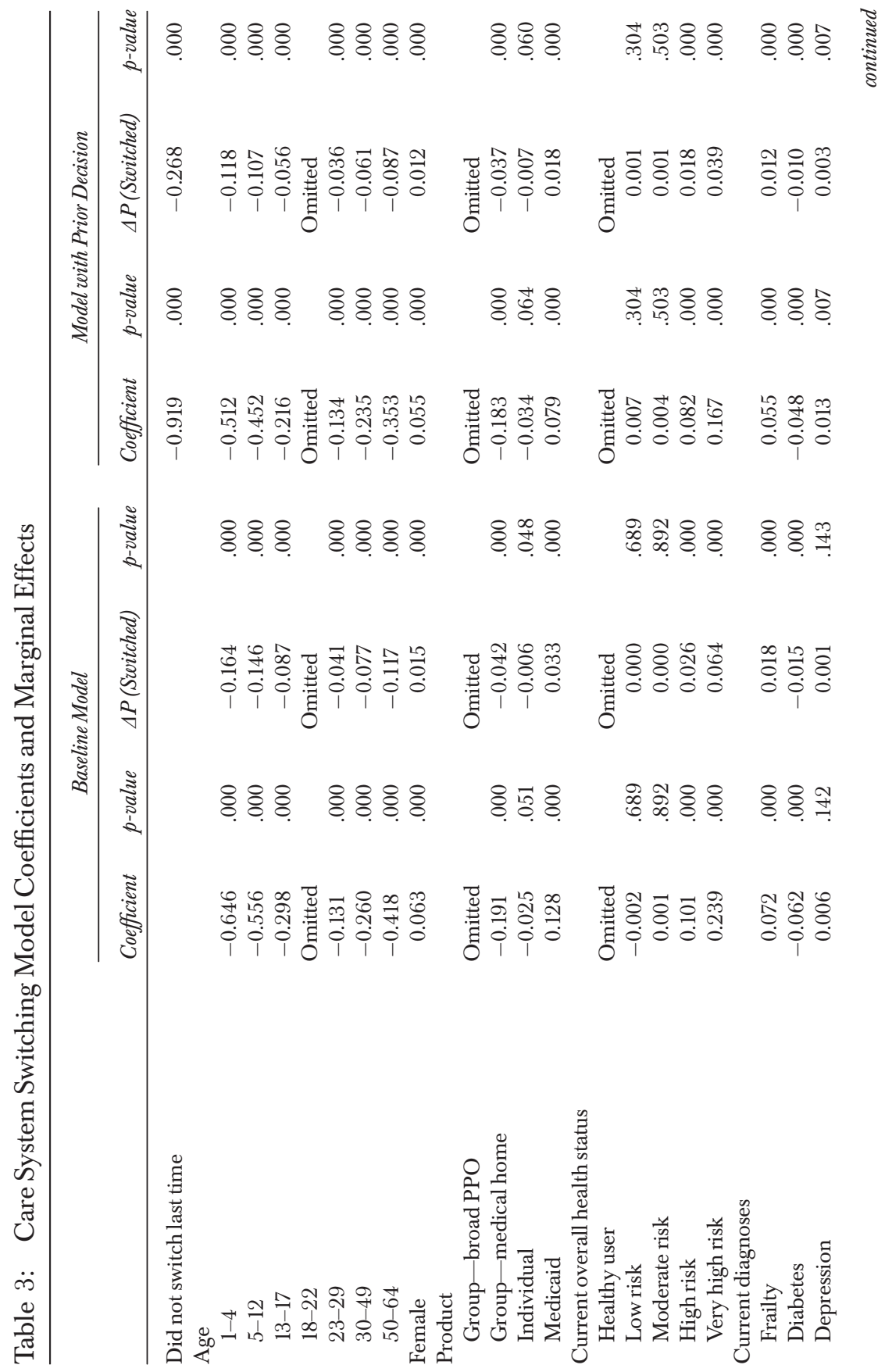




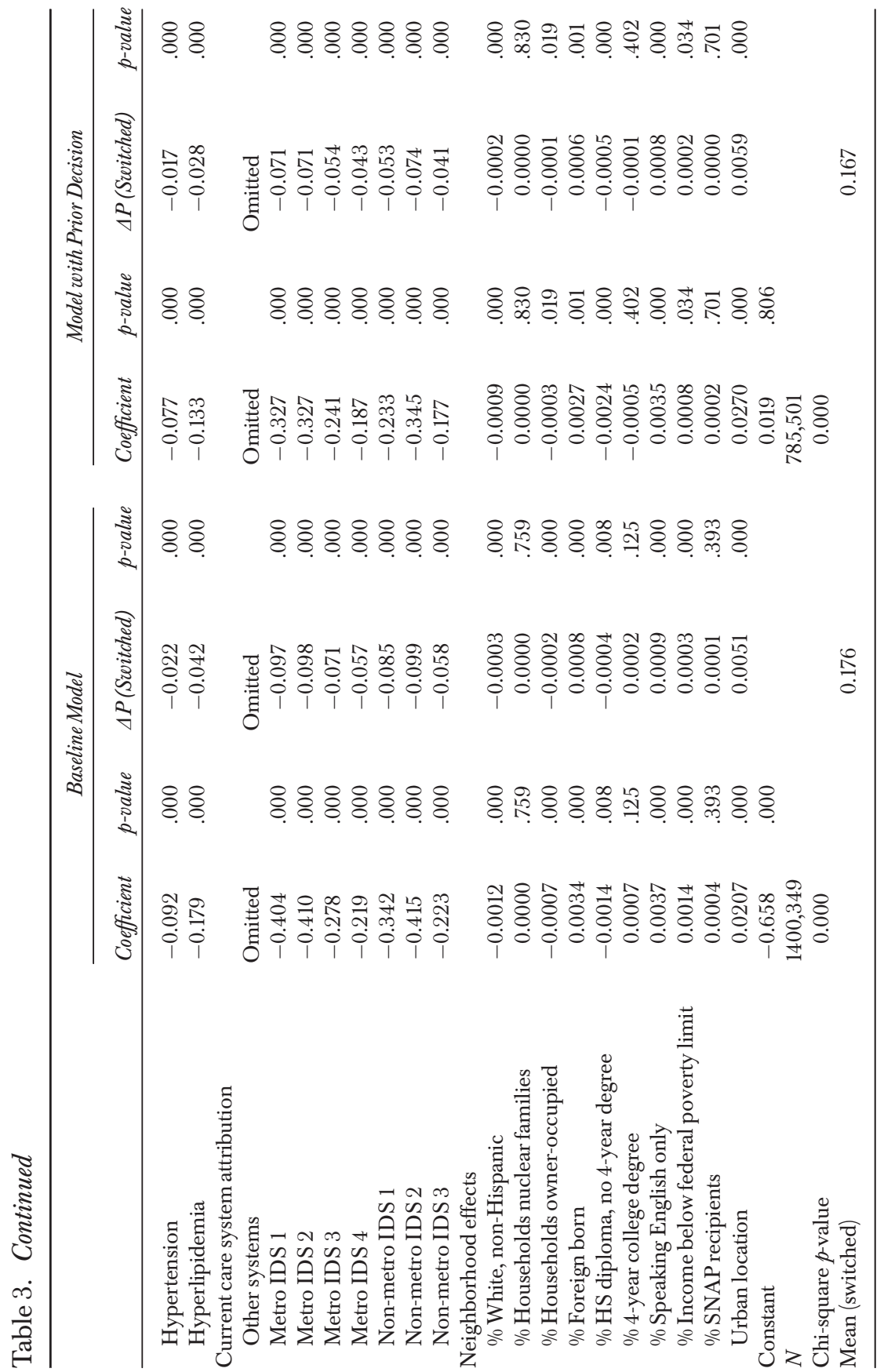


as we would expect to see a meaningful increase in stability of care system relationships when there is increased stability in the member's environment.

The factor with the greatest predictive power was past switching behavior, as seen in the second model in Table 3. Those who did not switch at the last opportunity had an average probability of switching that is a dramatic 26.8 percentage points lower, relative to those who did switch last time. It is interesting to note that the impact of adding this variable was primarily confined to the constant, with the relative magnitude and significance of the other marginal effects changing little.

\section{Duration of Patient Relationships with a Care System}

The duration model coefficients and marginal effects can be seen in Table 4, where marginal effects capture the change in median expected lifetime caused by a one-unit change in the percentage-based neighborhood effects, or by a change in binary and categorical variables from the reference value to the value in question. As suggested by the switching model, expected durations were shortest for those in the college-aged years (18-22) with marginal effects as great as +1.8 years for the category ages $5-12$. Women had a median expected duration that was 1.3 years shorter than a similar male. Unlike the switching model results, the impact of product follows expectations, most likely because the censoring controls allowed me to include a more representative individually insured population. Enrollment in a medical home product signaled tighter care system affiliation (marginal effect +1.1 years), and the transient nature of the individual and Medicaid populations was associated with shorter expected durations ( -1.6 and -1.3 years, respectively).

The impact of overall health status followed the patterns we saw in the switching model, showing a slight decline in expected duration for those at low risk relative to the healthy users ( -0.4 years), and durations steadily declining through the very high-risk members ( -1.8 years). Again, this is counter to expectations based on the literature, as we expect tighter care system affiliation when health status declines and frequency of visits presumably increase. As in the switching model, the impact of frailty and individual diagnoses is inconsistent, with marginal effects ranging from -0.4 to +0.4 years.

Relative to smaller care systems, an initial attribution to one of the large IDSs was associated with significant increases in the expected duration of patient affiliation with a care system. Marginal effects range from +0.6 to +2.4 years for these IDSs. It is noteworthy that there was a strong association between the size of the IDS and the magnitude of the marginal effect. 
Table 4: Weibull Duration Model Coefficients and Marginal Effects

\begin{tabular}{|c|c|c|c|c|}
\hline & Coefficient & $p$-value & $\Delta$ Median & $p$-value \\
\hline \multicolumn{5}{|l|}{ Age } \\
\hline $1-4$ & 0.287 & .000 & 1.636 & .000 \\
\hline $5-12$ & 0.318 & .000 & 1.843 & .000 \\
\hline $13-17$ & 0.115 & .000 & 0.601 & .000 \\
\hline $18-22$ & Omitted & & Omitted & \\
\hline $23-29$ & 0.024 & .019 & 0.117 & .091 \\
\hline $30-49$ & 0.156 & .000 & 0.832 & .000 \\
\hline $50-64$ & 0.255 & .000 & 1.433 & .000 \\
\hline Female & -0.221 & .000 & -1.301 & .000 \\
\hline \multicolumn{5}{|l|}{ Product } \\
\hline Group_-broad PPO & Omitted & & Omitted & \\
\hline Group-medical home & 0.163 & .000 & 1.086 & .000 \\
\hline Individual & -0.307 & .000 & -1.618 & .000 \\
\hline Medicaid & -0.241 & .000 & -1.312 & .000 \\
\hline \multicolumn{5}{|l|}{ Initial overall health status } \\
\hline Healthy user & Omitted & & Omitted & \\
\hline Low risk & -0.058 & .000 & -0.365 & .000 \\
\hline Moderate risk & -0.139 & .000 & -0.837 & .000 \\
\hline High risk & -0.174 & .000 & -1.032 & .000 \\
\hline Very high risk & -0.326 & .000 & -1.797 & .000 \\
\hline \multicolumn{5}{|l|}{ Initial diagnoses } \\
\hline Frailty & -0.067 & .000 & -0.382 & .001 \\
\hline Diabetes & -0.027 & .045 & -0.159 & .143 \\
\hline Depression & -0.075 & .000 & -0.427 & .000 \\
\hline Hypertension & -0.006 & .511 & -0.034 & .635 \\
\hline Hyperlipidemia & 0.070 & .000 & 0.422 & .000 \\
\hline \multicolumn{5}{|l|}{ Initial care system attribution } \\
\hline Other systems & Omitted & & Omitted & \\
\hline Metro IDS 1 & 0.363 & .000 & 2.182 & .000 \\
\hline Metro IDS 2 & 0.397 & .000 & 2.435 & .000 \\
\hline Metro IDS 3 & 0.271 & .000 & 1.554 & .000 \\
\hline Metro IDS 4 & 0.140 & .000 & 0.752 & .000 \\
\hline Non-metro IDS 1 & 0.110 & .000 & 0.580 & .000 \\
\hline Non-metro IDS 2 & 0.298 & .000 & 1.732 & .000 \\
\hline Non-metro IDS 3 & 0.114 & .000 & 0.602 & .000 \\
\hline \multicolumn{5}{|l|}{ Neighborhood effects } \\
\hline$\%$ White, non-Hispanic & 0.0003 & .113 & 0.0017 & .253 \\
\hline$\%$ Households nuclear families & 0.0003 & .172 & 0.0019 & .324 \\
\hline$\%$ Households owner-occupied & 0.0003 & .111 & 0.0018 & .251 \\
\hline$\%$ Foreign born & -0.0036 & .002 & -0.0210 & .022 \\
\hline$\%$ HS diploma, no 4-year degree & 0.0006 & .539 & 0.0034 & .658 \\
\hline$\% 4$-year college degree & -0.0022 & .008 & -0.0131 & .055 \\
\hline$\%$ Speaking English only & -0.0020 & .029 & -0.0118 & .114 \\
\hline$\%$ Income below federal poverty limit & -0.0009 & .082 & -0.0056 & .210 \\
\hline
\end{tabular}


Table 4. Continued

\begin{tabular}{lccrr}
\hline & Coefficient & p-value & AMedian & $p$-value \\
\hline \% SNAPrecipients & 0.0011 & .118 & 0.0066 & .260 \\
$\quad$ Urban location & -0.0049 & .590 & -0.0287 & .698 \\
Constant & 2.185 & .000 & & \\
Weibull shape parameter & 1.178 & .000 & & \\
$N$ & 433,297 & & & \\
Chi-square $p$-value & 0.000 & & 7.6 & \\
Mean (est. duration) & & & 5.9 & \\
Median(est. duration) & & & & \\
\hline
\end{tabular}

Only neighborhood effects related to the percentage foreign born and the percentage speaking only English show statistically significant marginal effects, but none of these neighborhood effects have great policy significance. The largest marginal effect suggests a -0.2 year decline in duration for a 10 percentage-point increase in the fraction of the census tract foreign born.

\section{Robustness of Results}

In this work, I used data from health plan members residing in the state of Minnesota. Because some of the more rural locations in Minnesota may have few alternative care systems, these residents may face constrained choices, and I may have over-estimated the loyalty of patients to their care systems when they have multiple options. For this reason, I re-estimated the models using only the subset of the population with an urban-focused Rural Urban Commuting Area code. We know there are multiple care system alternatives in these Twin Cities and Duluth metropolitan area census tracts. The parameter estimates were nearly identical to those presented here.

In addition, these results depend on the retrospective attribution method used by the health plan. It is possible that we would have different outcomes with different attribution methods. Unfortunately, this 6-year panel dataset does not contain the detailed claims needed to estimate alternative attribution. However, in related work (Higuera and Carlin, unpublished data) we explored the stability of attribution outcomes across a variety of attribution methods. We found surprisingly stable attribution when primary care claims are used for retrospective attribution, whether the method relies on all primary care services or evaluation and management visits only; whether based on allowed charges or number of encounters; and whether based on a majority or plurality of claims. Attribution is much less robust across methods which 
incorporate specialty care, and we would expect to see different outcomes in the work presented here if a combined primary and specialty care attribution method were the basis of measuring patient affiliation with a care system.

Finally, I chose to saturate the model by including both prior year health risk measures, as well as indicators of frailty or one of four diagnoses in the prior year. There may be a concern that this double-counts health status measures, leading to instability in the parameter estimates. I have re-estimated models excluding the frailty and specific diagnosis measures. The only meaningful change in parameter estimates was constrained to the parameters on the prior year overall health status measures, in patterns consistent with the original parameter estimates. In the attribution model, where the diagnosis parameters were positive, removing diagnosis indicators resulted in a steeper slope in the parameter estimates as the member had increasing health risk. In the switching duration model, where the diagnosis parameters were generally negative, removing diagnosis indicators caused a flattening of the parameter slope as health risk increased. In the duration model, the diagnosis parameters were small relative to health risk parameters, and there was a consistent small change in health risk parameters when the diagnosis indicators were removed.

\section{DISCUSSION}

In these results, we see significant levels of patient loyalty, with nearly half the patients' care system relationships projected to be longer than 6 years in duration (median expected relationship length: 5.9 years). In addition, once the member had shown loyalty to a care system, his or her chance of switching relationships was very low in future years. ${ }^{3}$ In this environment, investment in population health management makes sense for both the health of the member and the financial health of a care system in a shared savings contract.

It is also apparent that there was a significant reduction in the length of patient affiliation with a care system when the member has a high- or very high-risk health status. This is counterintuitive, as one would expect high-risk members to have strong loyalty to a care system due to more frequent interaction. However, when we consider that the measure of loyalty was based on primary care, and these high-risk members most likely had multiple specialty relationships - often across multiple care systems - this diffusion of loyalty makes sense. This also suggests an explanation for the reduced length of the patient's affiliation with a care system for women, who may have had their 
loyalty divided between a primary care physician and an OB/GYN in a separate practice. This theory is supported by the significant increase in the length of these relationships for members attributed to one of the seven largest integrated delivery systems, where it is more likely that loyalty was strengthened by primary care and specialty relationships being provided by the same care system. This suspected link between larger systems (implying co-located primary and specialty services) and increasing stability of patient affiliation with a care system adds a new dimension to the policy implications of provider consolidation.

\section{ACKNOWLEDGMENTS}

Joint Acknowledgment/Disclosure Statement: This work was funded with an intramural award from Medica Research Institute.

Disclosures: None.

Disclaimers: None.

\section{NOTES}

1. We recognize that those who choose the altruistic professions in health care are motivated by much more than financial reward when making treatment decisions for their patients. However, the financial viability of their practices is necessary to continuing to provide care.

2. Primary care was defined by place of service (office visits, or for those in the Medicaid product, emergency department visits) and the provider's specialty (internal medicine, general practice, family medicine, or OB/GYN).

3. The reader may be tempted to say that the finding that past loyalty predicts future loyalty is a tautology. However, this result provides important reassurance to the provider community that investment in population health for long-term patients should benefit their care systems in the future.

\section{REFERENCES}

Bailit, M., C. Hughes, M. Burns, and D. H. Freedman. 2012. "Shared Savings Payment Arrangements in Health Care: Six Case Studies." The Commonwealth Fund [accessed on August 28, 2012]. Available at http://www.commonwealthfund. org/ /media/Files/Publications/Fund\%20Report/2012/Aug/1624_Bailit_shared_savings_payment_arrangements_six_case_studies.pdf. 
Centers for Medicare and Medicaid Services. 2013. "Medicare Shared Savings Program” [accessed on May 10, 2013]. Available at http://www.cms.gov/Medicare/ Medicare-Fee-for-Service-Payment/sharedsavingsprogram/index.html

Christianson, J. B., D. Zismer, K. M. White, and J. Zeglin. 2011. Exploring Alternative Approaches to Valuing Physician Services. Report for the Medicare Payment Advisory Commission [accessed on August 28, 2012]. Available at http://medpac. gov/documents/Jun11_ValuingPhysicianServices_CONTRACTOR_AW.pdf.

Gallagher, N., A. MacFarlane, A. W. Murphy, G. K. Freeman, L. G. Glynn, and C. P. Bradley. 2013. "Service Users' and Caregivers' Perspectives on Continuity of Care in Out-of-Hours Primary Care." Qualitative Health Research 23 (3): $407-21$.

Guthrie, B. 2002. "Continuity in UK General Practice: A Multilevel Model of Patient, Doctor and Practice Factors Associated with Patients Seeing Their Usual Doctor." Family Practice 19 (5): 496-9.

Lee, J. H., Y. J. Choi, S. H. Lee, N. J. Sung, S. Y. Kim, and J. Y. Hong. 2013. "Association of the Length of Doctor-Patient Relationship with Primary Care Quality in Seven Family Practices in Korea." Journal of Korean Medical Science 28 (4): $508-15$.

Mehrotra, A., J. L. Adams, J. W. Thomas, and E. A. McGlynn. 2010. "The Effect of Different Attribution Rules on Individual Physician Cost Profiles." Annals of Internal Medicine 152 (10): 649-54.

Mendoza-Sassi, R., and J. U. Beria. 2003. "Prevalence of Having a Regular Doctor, Associated Factors, and the Effect on Health Services Utilization: A PopulationBased Study in Southern Brazil." Cadernos de Saúde Pública 19 (5): 1257-66.

Mohammed Al-Azri, M., and S. S. Ganguly. 2009. "Patients' Views of Interpersonal Continuity of Care in Four Primary Health Care Centres of Urban Oman.” Sultan Qaboos University MedicalJournal9 (3): 287-95.

Morris, D., and B. Eggbeer. 2011. "Total Cost of Care Contracts: Early Lessons." BDC Advisors Point of View [accessed on November 8, 2011]. Available at http://bdcadvisors.com/uploads/Total $\% 20$ Cost $\% 20$ of $\% 20$ Care $\% 20$ Contracts $\% 20$ Early $\%$ 20Lessons\%20June $\% 202011$.pdf.

Nutting, P. A., M. A. Goodwin, S. A. Flocke, S. J. Zyzanski, and K. C. Stange. 2003. "Continuity of Primary Care: To Whom Does It Matter and When?" Annals of Family Medicine 1 (3): 149-55.

Rosa Filho, L. A., A. G. Fassa, and V. M. Paniz. 2008. "Factors Associated with Interpersonal Continuity of Care: A Population-Based Study.” Cadernos de Saúde Pública 24 (4):915-25.

Saultz, J. W. 2003. "Defining and Measuring Interpersonal Continuity of Care." Annals of Family Medicine 1 (3): 134-43.

U.S. Census Bureau. 2012. "American Community Survey" [accessed on December 12, 2012]. Available at http://www.census.gov/acs/www/

Waibel, S., D. Henao, M. B. Aller, I. Vargas, and M. L. Vazquez. 2012. "What Do We Know about Patients' Perceptions of Continuity of Care? A MetaSynthesis of Qualitative Studies.” International Journal for Quality in Health Care 24 (1): 39-48. 
Weiner, J. P., B. H. Starfield, and R. N. Lieberman. 1992. "Johns Hopkins Ambulatory Care Groups (ACGs). A Case-Mix System for UR, QA and Capitation Adjustment." HMO Practice 6 (1): 13-9.

WWAMI Rural Health Research Center. 2013. "RUCA Map Classifications" [accessed on January 31, 2013]. Available at http://depts.washington.edu/uwruca/ruca-maps.php 